\section{Methodology for Reconstructing Early Zebrafish Development From In Vivo Multiphoton Microscopy}

Miguel A. Luengo-Oroz, Jose L. Rubio-Guivernau, Emmanuel Faure, Thierry Savy, Louise Duloquin, Nicolas Olivier,

David Pastor, Maria Ledesma-Carbayo, Delphine Débarre, Paul Bourgine, Emmanuel Beaurepaire, Nadine Peyriéras, and Andres Santos

\begin{abstract}
Investigating cell dynamics during early zebrafish embryogenesis requires specific image acquisition and analysis strategies. Multiharmonic microscopy, i.e., second- and third-harmonic generations, allows imaging cell divisions and cell membranes in unstained zebrafish embryos from 1- to 1000-cell stage. This paper presents the design and implementation of a dedicated image processing pipeline (tracking and segmentation) for the reconstruction of cell dynamics during these developmental stages. This methodology allows the reconstruction of the cell lineage tree including division timings, spatial coordinates, and cell shape until the 1000-cell stage with minute temporal accuracy and micrometer spatial resolution. Data analysis of the digital embryos provides an extensive quantitative description of early zebrafish embryogenesis.
\end{abstract}

Index Terms-Cell lineage tree, in vivo 4-D imaging, multiphoton microscopy, viscous watershed, zebrafish.

\section{INTRODUCTION}

The reconstruction of cells' clonal history is a long-standing goal of developmental biology, which involves being able to identify and digitalize each cell in time and space, leading to the cell lineage tree sequencing. In this context, image analysis is a critical step for understanding embryogenesis processes based on in vivo microscopy imaging observations [1]-[3]. The zebrafish, i.e., a vertebrate organism, is a reference model in the study of development, cancer, and stem cells [4]. Owing to the transparency of its tissues, it is possible to record high-resolution in vivo images of cell nuclei, cell

M. A. Luengo-Oroz, J. L. Rubio-Guivernau, D. Pastor, M. Ledesma-Carbayo, and A. Santos are with the Biomedical Image Technologies Laboratory of ETSI Telecomunicación, Universidad Politécnica de Madrid, 28040 Madrid, Spain, and also with Centro de Investigación Biomédica en Red Bioingeniería, Biomateriales y Nanomedicina (CIBER-BBN), Spain (e-mail: maluengo@die.upm.es).

E. Faure, T. Savy, and P. Bourgine are with the Centre de Recherche en Epistemologie Appliquee, Ecole Polytechnique, Centre National de la Recherche Scientifique, 75015 Paris, France.

L. Duloquin and N. Peyriéras are with the Neurobiologie et Developpement, Institut de Neurobiologie Alfred Fessard, Centre National de la Recherche Scientifique, 91198 Gif/Yvette, France.

N. Olivier, D. Débarre, and E. Beaurepaire are with Laboratory for Optics and Biosciences, Ecole Polytechnique, Centre National de la Recherche Scientifique, Institut National de la Santé et de la Recherche Médicale, 91128 Palaiseau, France. membranes, or reporters of gene expression activity during embryo development. The digital reconstruction of the first $24 \mathrm{~h}$ of zebrafish development has been previously drafted with light-sheet-based fluorescence microscopy [5]. Nevertheless, the quantitative description of embryos at early stages is impaired because the fluorescent protein expression at earliest stages is either weak or poorly localized in subcellular structures during the first hours post fertilization (hpf). By 3 hpf, the zebrafish zygote undergoes the midblastula transition (MBT) [6], which is characterized by cell cycle lengthening, loss of cell division synchrony, activation of transcription, and appearance of cell motility. Multiharmonic imaging is a valuable tool for the study of early zebrafish embryogenesis, since it does not need fluorescent markers [7], [8]. Hence, the acquisition of combined second- and third-harmonic generation (SHG and THG, respectively) imaging of unstained embryos provides a new 4-D description for the first ten divisions cycles. In this paper, we describe an ad hoc image processing and analysis methodology able to reconstruct the digital embryo from this new imaging modality. An earlier version of this paper was presented in [9].

\section{MULTIHARMONIC MiCROSCOPY}

Multiharmonic microscopy can be used to detect several key events during early embryo development [7], [8], [10]. THG signals are obtained from submicrometer inclusions such as lipid/water interfaces [11], and THG images of early zebrafish embryos reveal cell contours, yolk structure and dynamics, and the nuclear membrane during cell divisions. SHG is obtained from dense organized structures [12], revealing the dynamics of mitotic spindles (see Fig. 1). We performed a series of multimodal THG-SHG-two-photon excited fluorescence (2PEF) microscopy experiments in live H2B-mCherry zebrafish mutants expressing a red fluorescent protein in the nucleus in order to define the timing of THG/SHG signals with respect to chromatin dynamics and used that information for subsequent analysis in unstained embryos (see Fig. 2). We found that the SHG intensity from a dividing cell exhibits a nearly Gaussian temporal behavior permitting to measure division timing and cell cycle duration. We restricted the acquired volume by imaging the half-sphere containing the embryonic cells using an optimized conformal scanning scheme where each plane is scanned along a spiral trajectory with variable speed. This approach resulted in spending more time recording signal from the deepest parts of the embryo, where light scattering and aberrations decrease the signals, than at the periphery. In turn, this allowed minimizing both the acquisition time and the phototoxicity [8].

\section{OVERVIEW OF PROCESSING METHODS}

The goal of the proposed methodology is to write the individual history of each cell in terms of lineage and shape from the first to tenth cell cycles, going from 1 to 1024 cells ( $3 \mathrm{hpf}$ ). Input data are SHG and THG time-lapse 3-D images. The intracellular SHG signal exhibits a peak during mitosis that will be used to extract the lineage tree. The THG signal highlights membrane geometry and is used to segment the cell shape. A typical acquisition is in the order of 100 volumes of size $(512,512,120)$ with voxel size of $(2,2,4) \mu \mathrm{m}^{3}$ and $\Delta T=90 \mathrm{~s}$. The following context-specific features have been taken into account for designing the processing pipeline: 1) Visual observation shows that all mitosis during a cell cycle occur almost at the same time (e.g., all the cells from four to eight cells almost synchronously divide and then proceed as a wave through the embryo). 2) During this developmental period, we may assume that cells do not move except for mitosis. 3) During mitosis, daughter cells symmetrically move with respect to their mother cell. 


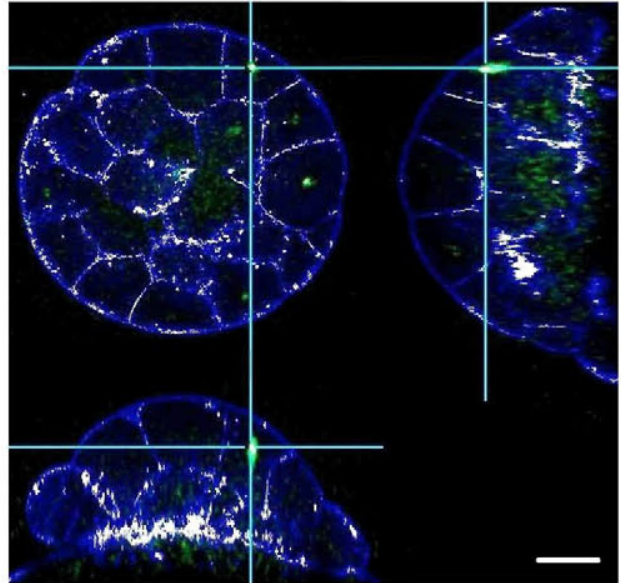

Fig. 1. Multiharmonic imaging of unstained zebrafish embryo at the 64-cell stage. (Green) SHG. (Blue-white) THG. (Scale bar is $100 \mu \mathrm{m}$, and the cross lines explicit the relative positions of the three cross sections.)
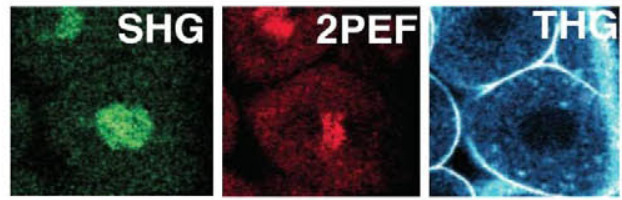

Fig. 2. Detail of THG-SHG-2PEF microscopy experiment in nuclei-stained zebrafish mutants showing a dividing cell. SHG reveals the unstained mitotic spindles. 2PEF reveals the mCherry-labeled chromatin. THG reveals the unstained cell contours.

Because errors in mitosis detection propagate along the lineage, the processing pipeline has been coupled with the MOVIT validation interface (Savy et al., unpublished). In the proposed procedure, divisions in each cell cycle are automatically detected and manually checked and corrected if needed before analyzing the next cell cycle. This strategy allows minimizing the human intervention to extract correct data. In Fig. 3, the processing pipeline is presented. First, the SHG image sequence is divided into subsequences corresponding to each cell cycle, and then, each subsequence is collapsed into one frame; thus, time lapses that typically have 100 time points are reduced to ten. Next, the lineage tree is extracted from collapsed SHG data (identifying the cell daughters of each cell in the next cycle). Then, SHG spot detection is manually corrected with the MOVIT interface, and the procedure of automatic detection and manual validation/correction is iterated for all the cycles. Next, the timing of mitosis events is refined using the noncollapsed original complete SHG sequence. Finally, the nucleus position of each cell is used as a seed for a viscous watershed segmentation of THG membrane images. In the following sections, all the steps are presented in detail.

\section{Processing Pipeline}

\section{A. SHG Cycle Fusion}

First, SHG data are filtered with a 3-D median operator and convolved with a Gaussian kernel for regularization purposes. Visual inspection of SHG data shows that the mitotic spots of each division cycle are organized in temporal waves across the embryo. The timing of this global mitotic clock can be extracted from the integration of the total SHG signal along time. The total-SHG-signal measurement presents an oscillatory behavior where the maxima correspond to the time points where most of the mitoses of a given division cycle happen and the

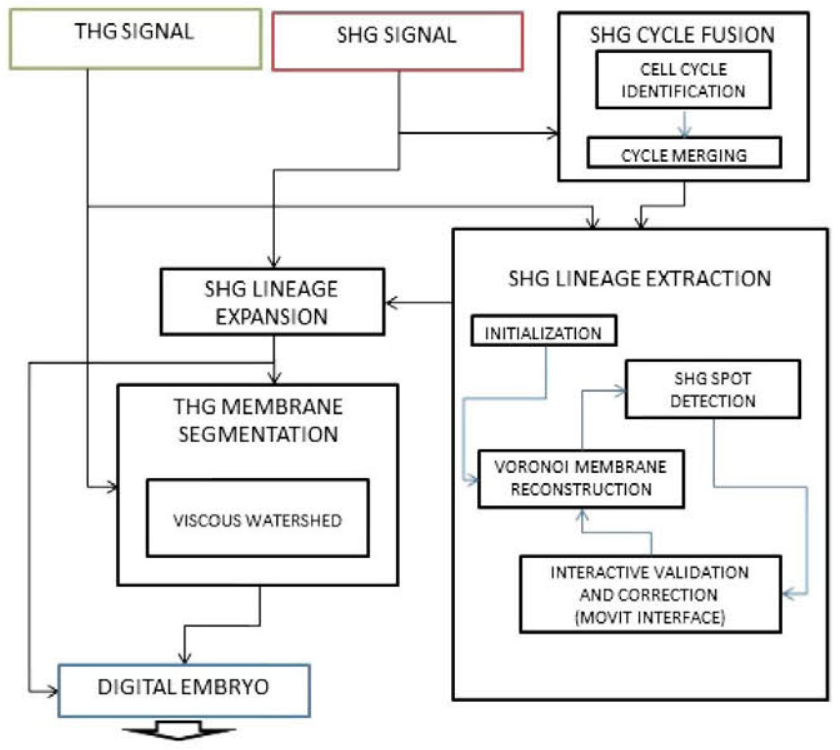

Fig. 3. Schema of the processing pipeline for generating the digital zebrafish embryo from SHG and THG data.

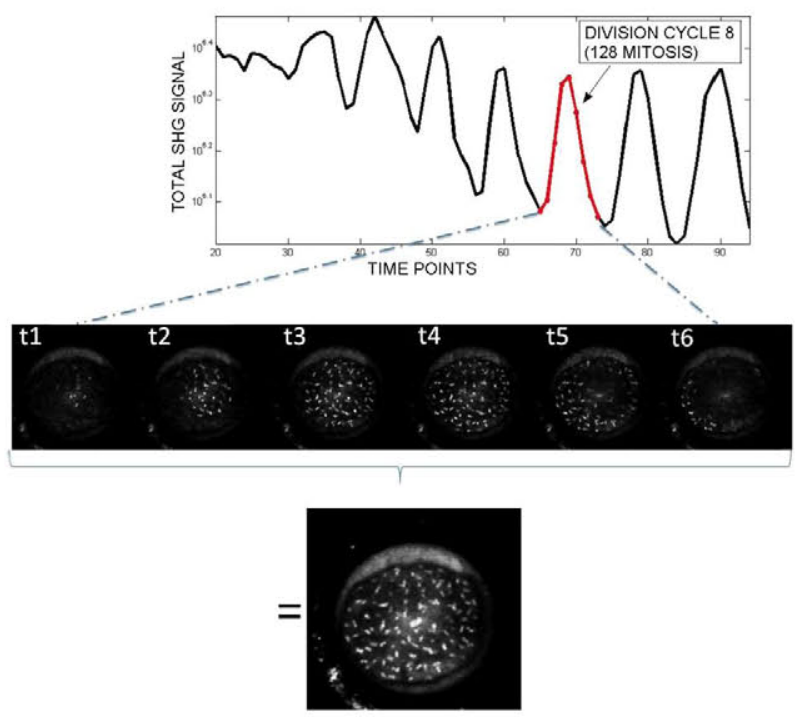

Fig. 4. Total SHG signal intensity along time (signal peaks correspond to division waves) and projection of consecutive SHG volumes and its resulting collapsed single volume (128-cell stage).

minima correspond to moments between two division waves. The identification of the function minima allows finding the time intervals between consecutive cell cycles. In order to produce a single 3 -D volume per division cycle, all the time points belonging to a division wave are gathered into a single 3-D image volume (see Fig. 4).

\section{B. SHG Lineage Extraction}

The goal of this module is to identify all the SHG spots in each division cycle and link each cell with its two daughter cells in the next cycle. The SHG spots are detected by finding image intensity maxima in a local neighborhood. The inputs of this module are the ten SHG-fused images corresponding to the first ten cycles and the ten whole embryo binary masks extracted from THG membrane data (the embryo masks are obtained by thresholding and then using a morphological close-hole operator to fill gaps inside the mask, and finally creating a morphological closing and an opening to smooth the result). The Voronoi regions 


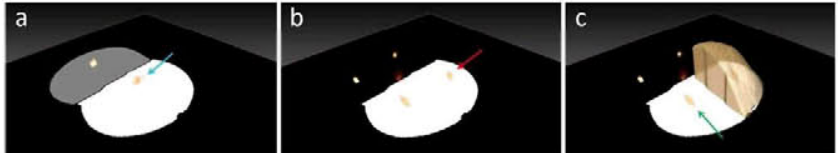

Fig. 5. Illustrative example of mitosis tracking. (a) Two-cell stage spots detection and associated Voronoi regions. Arrow points to the cell that is going to be tracked. (b) Red arrow points to the SHG spot detected in the four-cell stage inside (blue arrow) the Voronoi region of the previously pointed cell. (c) Green arrow points to the second daughter outside the isosurface region showing (red arrow) the forbidden region associated to the other daughter.

generated from cell centers inside the embryo mask are a reasonable approximation to membrane boundaries [13]. Hence, we can assume that, when a mother cell divides, the nuclei of the two daughter cells are going to appear inside the Voronoi region associated with their mother cell in the previous cycle. The iterative procedure has four steps.

1) Find the SHG spots corresponding to the first division cycle - two if starting with two cells. They are the two highest intensity regional maxima inside the embryo mask. Afterward, the Voronoi partition from the detected spots is created inside the embryo mask of the next division cycle.

2) Find the SHG spots corresponding to the next division cycle (twice the number of cells than that in the previous cycle). For each Voronoi region extracted from the previous cycle analysis, the maximum of intensity is found and corresponds to the first daughter cell. During mitosis, daughter cells have symmetric displacements relative to their mother cell; therefore, the search for the second daughter is restricted to the symmetric hemisphere inside the Voronoi region with respect to the first daughter and the mother cell. The local intensity maximum in this restricted region is selected as the second daughter cell (see Fig. 5).

3) The detected spots are visually validated and corrected using the MOVIT interface. Error rates in detection (coupled with tracking) before validation are in the order of $10 \% \pm 5 \%$ (depending on the data set and the cell cycle). Typical errors in SHG spot detection are due to the following reasons: 1) Low dynamic range of the SHG images and a granulated background, particularly next to the yolk, misleads the algorithm. 2) Other dense subcellular structures can be highlighted by SHG; occasionally, we detected additional tiny bright spots that might correspond to centrosomes. 3) At the end of cell division, mitotic spots appear as two-merged spots corresponding to the two groups of chromosomes. Depending on the temporal sampling along the whole mitosis, the contribution of the frame that contains this situation might be high in the collapsed image for the whole cycle. When this happens, combined with a particular geometrical disposition of the spot in relation to the cell mother nuclei, it is possible to detect both groups of chromosomes in the same spot as two dividing cells.

4) The Voronoi partition of the embryo mask in the next division cycle is created from detected spots. The procedure goes back to step 2 until all the cycles are analyzed.

At this point, keeping in mind that the goal is to obtain an error-free lineage, a comment should be made at the system level. Classical automated systems for cell lineage reconstruction first find cell centers and then link the cell mother and daughters a posteriori. Errors in center detection propagate and get amplified along the lineage; thus, even a small error rate in center detection leads to an inconsistent lineage. In addition, having little clue about the position of both false negatives and false positives makes the correction such a hard task because the validation and correction have to be made at a global level. In order to have an estimation of accuracy in spot detection on the volumes corresponding to the compressed lineage with a classical methodology, we applied a threshold followed by an area opening and a top-hat area opening (exhaustive parameters search). This methodology keeps structures of the right size above an intensity threshold, obtaining a success rate of $79 \%$ and $14 \%$ of false positives with an unknown distribution of their location. As an alternative to an automated system, manually extracting the cell lineage using a general-purpose annotation software, such as ITK Snap, is an almost impossible task (i.e., navigate inside a 3-D volume rendering where you should identify systematically hundreds of spots and link each one to a spot detected in the previous 3-D volume). When an experimented user uses a software designed for such a task, the lineage extraction can be manually achieved, but the need of expert work remains in the order of a hundred of hours (e.g., in order to reconstruct the complete lineage up to 512 cells, 1023 spots with 1022 links have to be created). Our strategy, which has the inherent benefit that produces the tracking coupled with the spot detection, consisted in doing an iterative manual correction based on contextual characteristics (number of spots and symmetry of cell division); the number of false positives is equal to the false negatives, meaning that correcting a spot simply consists in moving the spot position to a local neighborhood, which is easier and faster than creating or deleting spots. Using a simple maximum intensity to detect the spots is enough to keep the manual correction in the order of a couple of hours (very reasonable in the context of biological research). This method could be improved with more sophisticated spot detection methodologies, reducing the total time needed to the order of minutes; the utility of future automated systems should be based on a detection error rate tending to 0 or providing very controlled errors with an easy correction.

\section{SHG Lineage Temporal Expansion}

The previous module allowed the extraction of the cell lineage tree from the collapsed SHG images providing the parental relationships and the spatial positions. Nevertheless, it does not show the exact time of mitosis inside the division cycle. In order to achieve a better temporal resolution in the detection of SHG spots, the scanning time corresponding to each $x y$ slice in the conformal acquisition setup along the same volume has been taken into account.

Then, SHG intensity values are used to estimate mitosis times. The correlation between mitosis (anaphase, i.e., chromosome separation) and the maximum of the SHG signal intensity was verified in a threechannel (SHG, THG, and $2 \mathrm{PEF}$ ) experiment with $\Delta t=30 \mathrm{~s}$. In addition, we found that the SHG signal intensity during mitosis can be modeled as a Gaussian function (see in Fig. 6), whose maximum accurately approximates the temporal location of the maximum signal (error $=14.6 \mathrm{~s})$. When lower sampling rates are used (i.e., the whole embryo is imaged), using the maximum of intensity of the SHG signal in order to estimate mitosis timing gives increased error, and the use of Gaussian fitting improves accuracy. To verify this hypothesis, we performed the Gaussian fitting of the SHG signal intensity in three different subsampled versions of the previous $\Delta t=30 \mathrm{~s}$ experiment with a new $\Delta t=90 \mathrm{~s}$ (one out of three data points from the original sequence). As a result, the maximum intensity selection yields an error of $30 \pm 30 \mathrm{~s}$ with respect to the $\Delta t=30 \mathrm{~s}$ experiment, while the Gaussian fitting approach for detecting the mitosis timing has an error of just $14.5 \pm 4 \mathrm{~s}$ (see Fig. 6). In conclusion, the maximum of the fitted Gaussian function can be used to refine the temporal location of mitosis (see Fig. 7).

\section{THG Membrane Segmentation}

The THG signal serves as a membrane marker that allows extracting cell geometries. Cell segmentation is approached by a watershed-based methodology where the seeds are the cell centers extracted from the SHG spots. However, the THG signal delineating cell boundaries is poorly defined in some places, leading to leakage 

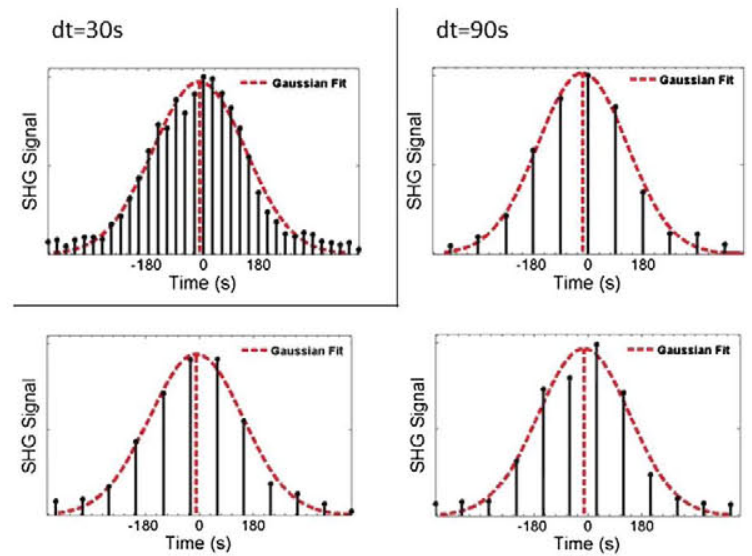

Fig. 6. SHG signal intensity profile during mitosis; chromosomes separate at $t=0$. The maximum of a Gaussian fitting of the SHG signal with $d t=30 \mathrm{~s}$ approximates mitosis with an error of $14.6 \mathrm{~s}$, whereas performing the Gaussian fitting in three different subsampled versions of $d t=90 \mathrm{~s}$ approximates the mitosis timing with an averaged precision of $14.5 \pm 4 \mathrm{~s}$.
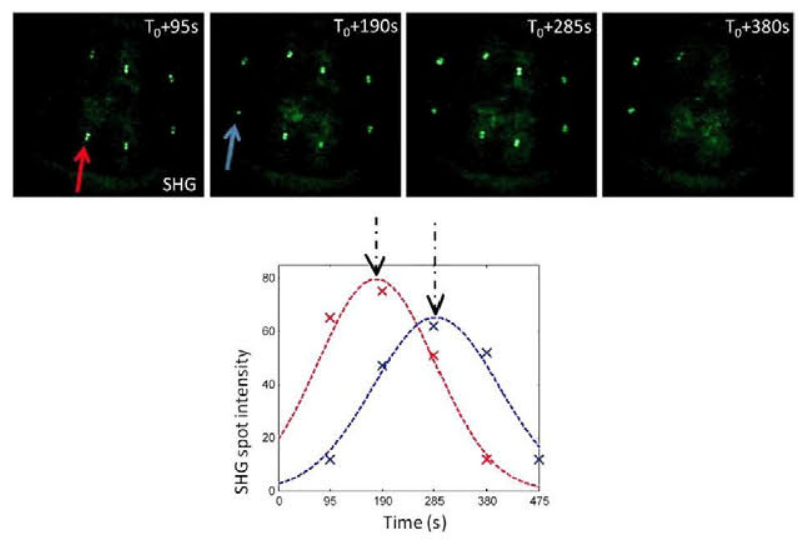

Fig. 7. (Top) Four consecutive frames of SHG signal. (Bottom) Intensity measurement at the different time steps on two cells and the Gaussian fitting of the SHG signal along time.

problems with the standard watershed. In order to address this issue, we have implemented a viscous watershed methodology [14] that consists in modifying the topology of the image by means of a viscous closing and then performing the standard watershed. This procedure is equivalent to performing a watershed with viscous fluid on the original landscape. The oil-model fluid has been chosen for the viscous closing transformation (see Fig. 8). In this model, the simulated temperature is associated to the intensity level $h$. Oil at low temperatures (dark pixels) has high viscosity so that fluid does not leak; with high temperature (white pixels), oil is less viscous, and watershed lines follow the relief. The viscous closing transformation $T(f)$ may be implemented by adding $(V)$ the binary closings $\varphi$ of image $f$ at all the intensity levels $h>0$ with a ball of radius $r(h)$, i.e., $T(f)=\vee_{h>0} \varphi_{r(h)}\left(h \cdot \chi_{h}(f)\right)$ with $\chi_{h}(f)(p)=1$ for all voxels $p$, where $f(p) \geq h$ and $\chi_{h}(f)(p)=0$ elsewhere. Function $r(h)$ determines the behavior of the fluid at different levels and is defined by the oil-model function $r(h)=R_{\max }-h * R_{\text {step }}$ performed for $r(h)>0$. Parameter $R_{\max }$ has been empirically chosen, and tests showed that the best results are achieved when $R_{\max }$ is around six times smaller than the estimated mean cell radius at the corresponding cell cycle and $R_{\text {step }}$ has a fixed value $R_{\text {step }}=R_{\max } / h_{v}$, related to level $h_{v}$, i.e., the intensity maximum of the THG signal. In order to

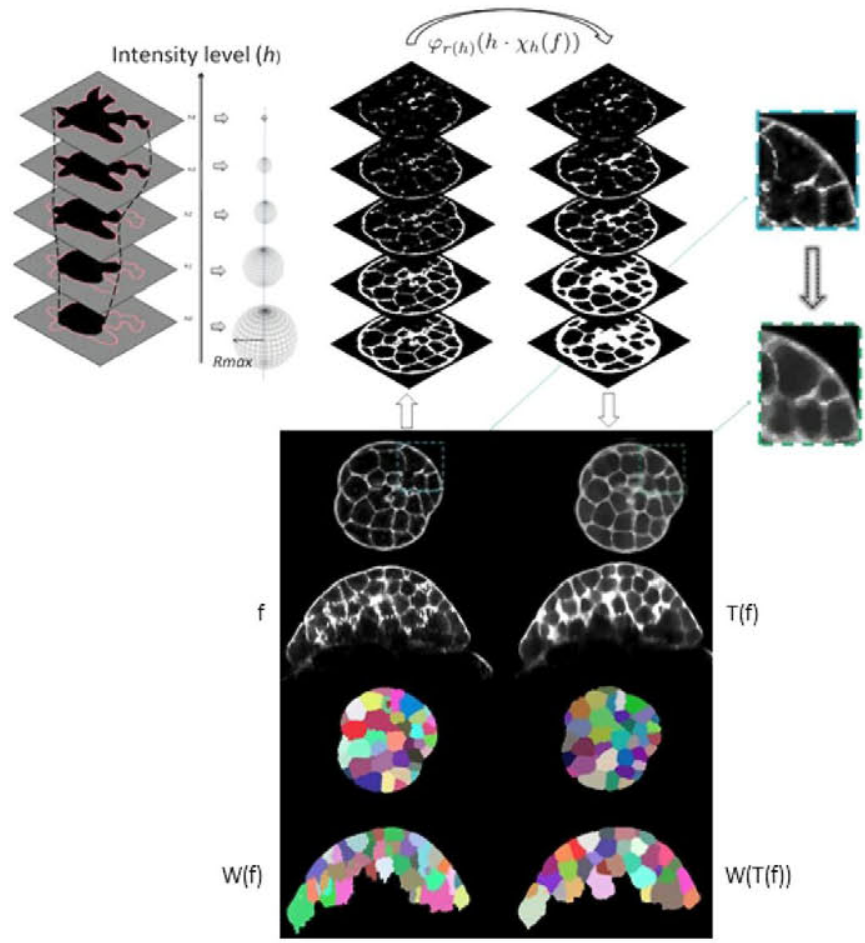

Fig. 8. Viscous closing procedure and example of THG membrane segmentation. T(f): viscous closing; f: raw data; W(f): watershed; W(T(f)): viscous watershed.

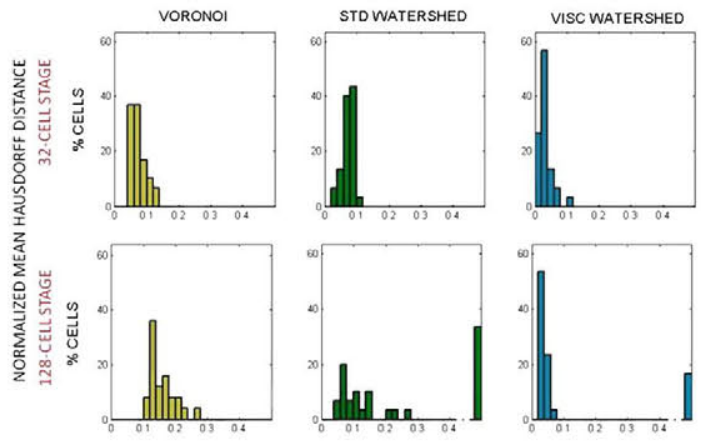

Fig. 9. Histograms showing the percentage of cells at the 32- and 128-cell stage regarding the relative mean Hausdorff distance when comparing the gold standard with the Voronoi region, the standard, and the viscous watershed segmentation (columns in the extreme right show segmented cells whose evaluation is bigger than 0.5 , undersegmented or oversegmented).

assess the segmentation quality against a manually segmented gold standard, which is obtained using ITK Snap [15], a variant of the Hausdorff distance [16] has been calculated for each cell in different division cycles. We define the relative mean Hausdorff distance as the mean of all the minimal Euclidian distances calculated from each of the voxels of the segmented membrane surface to the gold standard cell surface and the result divided by the radius of a spherical cell with the same volume as the gold standard cell, e.g., for two concentric spheres with double radius, the relative mean Hausdorff distance value is 0.5. While the Voronoi region provides a robust rough approximation, the viscous watershed segmentation is more accurate and produces less leakage than the standard watershed (see Fig. 9). The main source of errors comes from the leakage produced by the bottom cells that are in the interphase with the yolk. 
embryo1
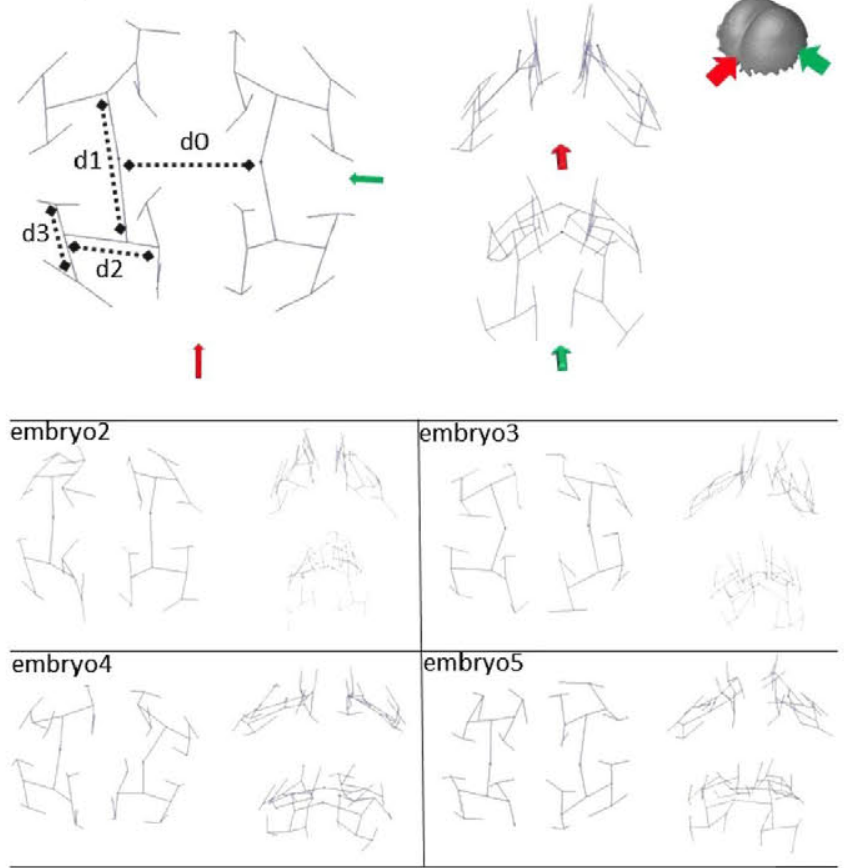

Fig. 10. Spatial deployment of the cell lineage tree for five embryos from 1to 32-cell stage. Mitosis alternatively occur along two perpendicular axes referenced by the green and red arrows until the 16-cell stage. Dipoles $d_{k}$ show the displacement of cell nuclei in mitosis at cycle $k$, where $d_{0}$ is the distance between the first two nuclei after the division of the zygote.

\section{RESULTS}

The presented processing pipeline provides the cell lineage tree annotated with the cell positions in each time step (corresponding to the nuclei position where the SHG spot signals the mitotic state), the parental relationship between cells, and the cell shape calculated both with the Voronoi approximation and the viscous watershed segmentation of the THG signal. Reconstructed data has been organized into an Oracle database and can be interactively visualized. Methods have been written in MATLAB, and the whole process for analyzing a complete data set takes about $72 \mathrm{~h}$ of computation (Core2Duo, 8-Gb random access memory) including $4 \mathrm{~h}$ of user interaction for manual validation and script launching distributed along the process (each cell cycle has to be validated before processing the following one). Six zebrafish embryos have been completely reconstructed from a two-cell stage to the 1000 -cell stage. A detailed description of the biological events occurring can be found in [8]; hereafter, we report some quantitative descriptions that can be used for further mathematical modeling purposes.

- The spatial distribution of mitotic spots during the first cell cycles shows a prototypical fractal-like structure for the six embryos analyzed (see Fig. 10). Mitosis alternatively occurs along two perpendicular axes until the 16-cell stage. Displacement $d_{k}$ of cell nuclei in mitosis at cycle $k$ is $\sim 0.85 d_{k-1}$, where $d_{0}=245 \pm 20 \mu \mathrm{m}$ is the distance between the first two nuclei. In the division from 16 to 32 cells, cells do not have enough physical space to divide in the same plane following the previous rule. The four centered cells divide toward the center of the embryo allowing the formation of a partially double-layered blastoderm.

- We found an interembryonic variability of developmental speed around $10 \%$. However, all the six embryos analyzed showed a similar behavior concerning division timings and cell cycle length. Interestingly, the mean cell cycle length perfectly fits with a parabolic function with the vertex between 32 and 64 cell

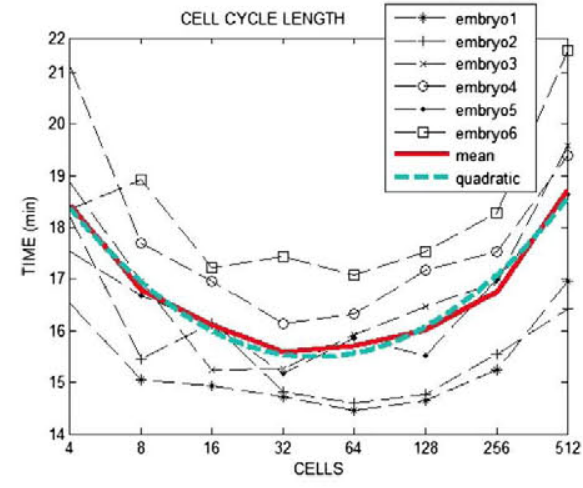

Fig. 11. Mean cell cycle duration from cycles 2 to 10 for six embryos. (Red) Average and (blue) quadratic fitting.

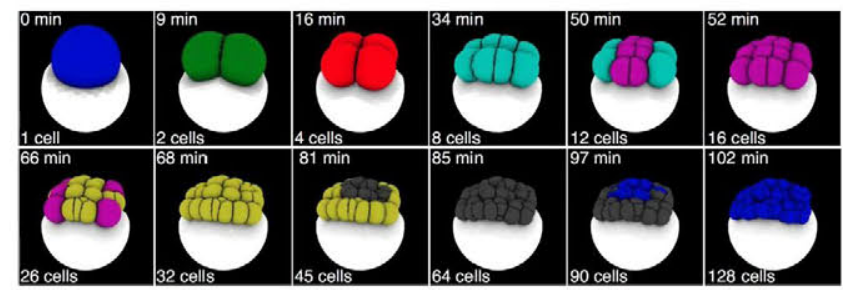

Fig. 12. Prototypical embryo development obtained from the spatiotemporal alignment of six embryos. Each color represents a new cell cycle.

stages (see Fig. 11), and is correlated with plasma membrane completion between the lower cells and the yolk. Therefore, the four-cell cycle length is the same as the 512-cell cycle length; 8 and 256-cell cycle lengths are equivalent and so are 16 and 128 , and 32 and 64 . We may conjecture that the two-cell-stage cycle length coincides with the 1000-cell cycle length. At this cycle (1000), cells are in the middle of the MBT regime conversion, cells become motile, and transcription occurs.

- A prototypic embryo has been modeled by superposing the lineage trees and averaging cell positions, division times, and cell division angles during mitosis. This prototype allows characterizing a normal embryo (see Fig. 12) during this developmental stage, where cells continuously drift out of synchrony and the cell cycle lengthens according to cell radial position, leading to division wave patterns across the embryo.

\section{MEthod Limitations AND FUtURE WorK}

The previous image processing methodology is limited to the analysis until 1000-cell stage because, later on, cells enter a developmental period characterized by mitosis desynchronization and random mitotic patches over the embryo. Furthermore, fast and oriented cell movements begin, and a row of cells is formed inside the yolk lacking of cell membranes (yolk syncytial layer). However, combined SHG-THG can be also used to image later stages of development (e.g., SHG reveals muscle structures) and other living-animal models. The methodology presented in this paper is an ad hoc system devoted to solve a specific problem, and the quantitative biological descriptions obtained from our pipeline could be also incorporated as a posteriori contextual information to design a better solution. In the near future, more processing methodologies should be adapted for detecting SHG spots [17]. We also hypothesize that new processing strategies involving image registration techniques for obtaining the displacement vector field of THG data should be designed to perform the lineage reconstruction of later developmental stages from such data. In order to improve THG membrane segmentation, a method that models the cell size and shape, as 
well as temporal redundancy, should be required. Another interesting analysis perspective can be to directly analyze the $3 D+t$ data as a $4-\mathrm{D}$ volume both for SHG and THG. In summary, in vivo SHG and THG microscopy represents a new powerful bioimaging modality and new processing methodologies have to be developed in consonancy [18].

\section{CONCLUSION}

In this paper, a dedicated image acquisition and processing pipeline for tracking and segmentation of SHG/THG image sequences of early zebrafish development has been designed and implemented. This methodology has been used for obtaining the digital reconstruction of six embryos including complete division timings, cell coordinates, and cell shape until the 1000-cell stage with minute temporal accuracy and micrometer spatial resolution. The data analysis of the reconstructed embryos provide a new comprehensive quantitative description of early zebrafish development [8].

\section{REFERENCES}

[1] A. Oates, N. Gorfinkiel, M. González-Gaitán, and C.-P. Heisenberg, "Quantitative approaches in developmental biology," Nat. Rev. Genet. vol. 10 , no. 8, pp. 517-530, Aug. 2009.

[2] C. Zanella, M. Campana, B. Rizzi, C. Melani, G. Sanguinetti, P. Bourgine, K. Mikula, N. Peyrieras, and A. Sarti, "Cells segmentation from 3-D confocal images of early zebrafish embryogenesis," IEEE Trans. Image Process., vol. 19, no. 3, pp. 770-781, Mar. 2010.

[3] M. Luengo-Oroz, M. Ledesma-Carbayo, N. Peyriéras, and A. Santos, "Image analysis for understanding embryo development: A bridge from microscopy to biological insights," Curr. Opin. Genet. Dev., vol. 21, no. 5, pp. 630-637, Oct. 2011.

[4] J. Amatruda, J. L. Shepard, H. M. Stern, and L. I. Zon, "Zebrafish as a cancer model system," Cancer Cell, vol. 1, no. 3, pp. 229-231, Apr. 2002.

[5] P. Keller, A. D. Schmidt, J. Wittbrodt, and E. H. K. Stelzer, "Reconstruction of zebrafish early embryonic development by scanned light sheet microscopy," Science, vol. 322, no. 5904, pp. 1065-1069, Nov. 2008.

[6] D. Kane and C. B. Kimmel, "The zebrafish midblastula transition," Development, vol. 119, no. 2, pp. 447-456, Oct. 1993.

[7] C. Sun, S. W. Chu, S. Y. Chen, T. H. Tsai, T. M. Liu, C. Y. Lin, and H. J. Tsai, "Higher harmonic generation microscopy for developmental biology," J. Struct. Biol., vol. 147, no. 1, pp. 19-30, Jul. 2004.

[8] N. Olivier, M. A. Luengo-Oroz, L. Duloquin, E. Faure, T. Savy, I. Veilleux, X. Solinas, D. Débarre, P. Bourgine, A. Santos, N. Peyriéras, and E. Beaurepaire, "Cell lineage reconstruction of early zebrafish embryos using label-free nonlinear microscopy," Science, vol. 329, no. 5994, pp. 967-971, Aug. 2010.

[9] M. Luengo-Oroz, T. Savy, J. L. Rubio, L. Duloquin, E. Faure, N. Olivier, M. Ledesma-Carbayo, D. Debarre, P. Bourgine, E. Beaurepaire, N. Peyrieras, and A. Santos, "Processing pipeline for digitalizing the lineage tree of early zebrafish embryogenesis from multiharmonic imaging," in Proc. 8th IEEE Int. Symp. Biomed. Imag., 2011, pp. $1561-1564$.

[10] D. Débarre, W. Supatto, E. Farge, B. Moulia, M. C. Schanne-Klein, and E. Beaurepaire, "Velocimetric third-harmonic generation microscopy: Micrometer-scale quantification of morphogenetic movements in unstained embryos," Opt. lett., vol. 29, no. 24, pp. 2881-2883, Dec. 2004.

[11] D. Débarre, W. Supatto, A. M. Pena, A. Fabre, T. Tordjmann, L. Combettes, M. C. Schanne-Klein, and E. Beaurepaire, "Imaging lipid bodies in cells and tissues using third-harmonic generation microscopy," Nat. Methods, vol. 3, no. 1, pp. 47-53, Jan. 2006.

[12] W. Zipfel, R. M. Williams, R. Christie, A. Y. Nikitin, B. T. Hyman, and W. W. Webb, "Live tissue intrinsic emission microscopy using multiphoton-excited native fluorescence and second harmonic generation," Proc. Nat. Acad. Sci., vol. 100, no. 12, pp. 7075-7080, Jun. 2003.

[13] M. Luengo-Oroz, L. Duloquin, C. Castro, T. Savy, E. Faure, B. Lombardot, R. Bourgine, N. Peyrieras, and A. Santos, "Can Voronoi diagram model cell geometries in early sea-urchin embryogenesis?," in Proc. 5th IEEE Int. Symp. Biomed. Imag., 2008, pp. 504-507.

[14] C. Vachier and F. Meyer, "The viscous watershed transform," Math. Imag. Vis., vol. 22, no. 2/3, pp. 251-267, May 2005.
[15] P. A. Yushkevich, J. Piven, H. C. Hazlett, R. G. Smith, S. Ho, J. C. Gee, and G. Gerig, "User-guided 3-D active contour segmentation of anatomical structures: significantly improved efficiency and reliability," Neurolmage, vol. 31, no. 3, pp. 1116-1128, Jul. 2006.

[16] D. Huttenlocher, G. A. Klanderman, and W. J. Rucklidge, "Comparing images using the Hausdorff distance," IEEE Trans. Pattern Anal. Mach. Intell., vol. 15, no. 9, pp. 850-863, Sep. 1993.

[17] I. Smal, M. Loog, W. Niessen, and E. Meijering, "Quantitative comparison of spot detection methods in fluorescence microscopy," IEEE Trans. Med. Imag., vol. 29, no. 2, pp. 282-301, Feb. 2010.

[18] D. Evanko, "Microscope harmonies," Nat. Methods, vol. 7, no. 10, p. 779 , Oct. 2010. 